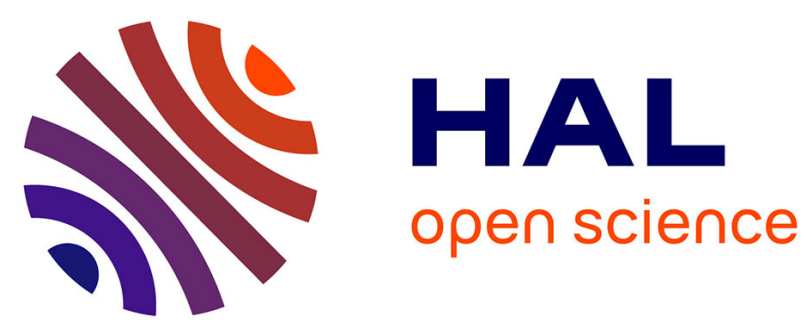

\title{
Predominance of the second-order, two-step mechanism in the electron impact double ionization of helium at intermediate impact energy
}

A Lahmam-Bennani, E M Staicu Casagrande, A Naja, C Dal Cappello, P Bolognesi

\section{To cite this version:}

A Lahmam-Bennani, E M Staicu Casagrande, A Naja, C Dal Cappello, P Bolognesi. Predominance of the second-order, two-step mechanism in the electron impact double ionization of helium at intermediate impact energy. Journal of Physics B: Atomic, Molecular and Optical Physics, 2010, 43 (10), pp.105201. 10.1088/0953-4075/43/10/105201 . hal-00569788

\section{HAL Id: hal-00569788 \\ https://hal.science/hal-00569788}

Submitted on 25 Feb 2011

HAL is a multi-disciplinary open access archive for the deposit and dissemination of scientific research documents, whether they are published or not. The documents may come from teaching and research institutions in France or abroad, or from public or private research centers.
L'archive ouverte pluridisciplinaire HAL, est destinée au dépôt et à la diffusion de documents scientifiques de niveau recherche, publiés ou non, émanant des établissements d'enseignement et de recherche français ou étrangers, des laboratoires publics ou privés. 


\title{
Predominance of the second order, two-step mechanism in the electron impact double ionization of Helium at intermediate impact energy
}

\author{
A Lahmam-Bennani ${ }^{1,2}$, E M Staicu Casagrande ${ }^{1,2}$, A Naja ${ }^{1,2,5}$, C Dal Cappello ${ }^{3}$ and P \\ Bolognesi $^{4}$ \\ ${ }^{1}$ Université Paris-Sud 11, Institut des Sciences Moléculaires d'Orsay (ISMO), Bât. 351, \\ 91405 Orsay Cedex, France \\ ${ }^{2}$ CNRS-ISMO, Bât. 351, 91405 Orsay Cedex, France \\ ${ }^{3}$ Université Paul Verlaine-Metz, Laboratoire de Physique Moléculaire et des Collisions, \\ ICPMB (FR 2843), Institut de Physique, 1 rue Arago, 57078 Metz Cedex 3, France \\ ${ }^{4}$ CNR-IMIP, Area della Ricerca di Roma 1, CP 10, 00016 Monterotondo Scalo, Italy \\ e-mail : azzedine.bennani@u-psud.fr
}

PACS 34.80.Dp (also 34.50.Fa and 34.80.-i)

\begin{abstract}
The (e,3-1e) four-fold differential cross sections (4DCS) are measured for the double ionization of helium in coplanar asymmetric geometry for a wide range of ejected electron energies and at an incident energy of about $600 \mathrm{eV}$. The experimental angular distributions of the 4DCS are characterized by large angular shifts of the forward and backward lobes with respect to the momentum transfer direction or its opposite, respectively. This validates our previously published results [Lahmam-Bennani et al $2002 \mathrm{~J}$. Phys. B: At. Mol. Opt. Phys. 35 L59] which were questioned by Götz et al [2003 J. Phys. B: At. Mol. Opt. Phys. 36 L77]. A qualitative, kinematical analysis is given which allows relating these shifts and the observed structures in the intensity distributions to the second order, 'two-step 2' double ionization mechanism, which is shown to predominate over the first-order 'shake-off' and 'two-step 1' mechanisms under the present kinematics.
\end{abstract}

\footnotetext{
${ }^{5}$ Permanent address: Lebanese University, Faculty of Science 3, Physics Department, Tripoli, Lebanon
} 


\section{Introduction}

The study of multiple ionization processes by charged particle impact is of considerable interest in many branches of physics, such as astrophysics or plasma physics, and also in other sciences such as life sciences where it is of prime importance for instance to understand the various mechanisms leading to energy deposition by radiation in matter. Electron impact double ionization (DI) is one of the simplest and the most fundamental of such processes. The advent of multi-parameter detection techniques, together with the progress in computational methods, has made it possible to perform complete experiments in which all kinematical parameters (vector momenta and energies) of all involved particles are determined. These are the so-called (e,3e) experiments, which have been successfully used during the last two decades to investigate in very fine details the ionization process on a variety of atomic targets, see [1-22] and references therein. However, $(e, 3 e)$ experiments remain difficult to perform because they necessitate the triple coincidence detection of the three final state electrons [1] (or alternatively the residual ion plus two electrons [15]). Besides, the corresponding cross section is inherently very small due to it's multiply (five-fold) differential character, consequently resulting in relatively modest statistics for the obtained data. It is therefore also of interest to consider (e,3-1e) experiments where, with respect to the (e,3e) case, one electron is not detected, hence only necessitating a double coincidence experiment similar to an $(\mathrm{e}, 2 \mathrm{e})$ single ionization (SI) but with energetics corresponding to DI. These experiments were first introduced by Lahmam-Bennani and coworkers (23-26) and further developed in [27-31]. In all these works, it was clearly demonstrated that (e,3-1e) experiments provide a very sensitive mean to identify the various mechanisms responsible for electron impact DI and to gauge their relative importance.

Direct electron impact DI may proceed mostly via three routes [32-33]. (i) In the shakeoff mechanism (SO), the incident electron interacts only once with a single target electron which is ejected without further interaction with other target electrons. Subsequent ejection of a second electron occurs, due to the electronic relaxation caused by the sudden change in the distribution of potential seen by this electron. (ii) In the two-step mechanism termed TS1, the projectile electron interacts with one target electron which subsequently collides with a second electron leading to ejection of the pair. (iii) In the two-step mechanism termed TS2, the projectile electron successively collides with two target electrons, resulting in their double ejection. Both SO and TS1 involve only one single interaction of the projectile with the target, hence their denomination as first order or first Born mechanisms (in the Born series). In 
contrast, TS2 involves two successive projectile - target interactions and hence is a second order or second Born mechanism. The most obvious signature of the presence of these mechanisms is that first order mechanisms yield ejected electron(s) angular distributions which are symmetrical with respect to the momentum transfer vector direction, whereas this symmetry is broken in the case of second order, TS2 mechanism, see [34,35]. LahmamBennani and coworkers $[8,9,12]$ reported the observation of non-first order effects in their measurements of fully differential $(\mathrm{e}, 3 \mathrm{e})$ cross sections for DI of Helium. As one would expect [36], these effects were found to be small at $5.5 \mathrm{keV}$ impact energy [8] while they become increasingly stronger as the energy is decreased to $1.1 \mathrm{keV} \mathrm{[9]} \mathrm{and} \mathrm{to} \sim 600 \mathrm{eV} \mathrm{[12].}$ Similar non-first order effects were also reported in [18] for (e,3e) DI of He at $500 \mathrm{eV}$ impact energy. In the low energy work of Lahmam-Bennani et al [12], the experimental angular distributions displayed large shifts of the forward peak from the momentum transfer direction, which could only partially be reproduced by second-Born calculations.

Similar large shifts were also observed in the (e,3-1e) experiments on DI of $\mathrm{He}$ and $\mathrm{H}_{2}$ at $\sim 600 \mathrm{eV}$ [29]. A tentative description of this latter work on $\mathrm{H}_{2}$ was developed by Mansouri et al [37] using a second-Born treatment (and correlated single center wavefunctions) which includes the double interaction between the incoming electron and the target. The results were able to produce only part of the shift of the experimental peaks. A more recent theoretical investigation using the external complex scaling method in spheroidal coordinates [38] also failed to describe the experimental findings for $\mathrm{He}$ as well as for $\mathrm{H}_{2}$. The authors concluded that the disagreement of theory with experiment is caused by the sequential TS2 mechanism of DI. On the other hand, a tentative description of the He (e,3-1e) results published in [29] was given by Götz et al [39,40] where the four-particle continuum was described in different approximations leading up to a $6 \mathrm{C}$ wavefunction which takes all two-body interactions into account as well as the interactions between each outgoing electron and the nucleus, and hence goes beyond the second Born approximation. However, the authors could not reproduce the strong asymmetry seen in the measured data, so that they came to implicitly question the experiments by writing [39]: <<we suggest that it is very important to establish the validity or otherwise of the asymmetry in the experimental data $>>$.

Given this situation and this questioning, we decided to reexamine the problem by taking advantage of the extended capabilities and sensitivity of our spectrometer gained by including to it new developments described in [41]. Our aim is to produce more extensive experimental data which would validate or invalidate the observations made in [29] and against which the theoretical predictions can be tested. To this end, we report here new (e,3-1e) experiments for 
DI of He performed at about the same impact energy of $\sim 600 \mathrm{eV}$ as in [29]. Besides, different energy sharings between the ejected electrons are investigated, in order to test the sensitivity of the results to this energy sharing, and to address the question whether features which appear very pronounced in the measured distributions and which are attributed to TS2 mechanism may strongly depend (or not) upon the difference in energy of the outgoing electrons. In the absence of elaborate non-first order theoretical calculations, the experimental data are compared with the results of a first-order theoretical model, namely the BBK model [42] which has proved to successfully describe experiments of this type in the few keV energy range [43]. Such comparison allows visualizing the strong shifts observed in the experimental data. Moreover, a qualitative discussion based on simple kinematical arguments is presented which shows that the observed large shifts can be related to the presence of TS2 mechanism which dominates over SO and TS1 mechanisms.

\section{Experiment}

The experimental set-up and experimental procedure used for the present work are described in detail in [41]. The main characteristic of the spectrometer is the unique combination of three high-efficiency, multi angle toroidal electrostatic energy analysers. Briefly, an incident electron beam with energy $E_{0}$ collides with the gas jet formed at the collision centre. A coplanar geometry is used, where all electrons are observed in the collision plane defined by the incident and scattered momentum vectors, $\boldsymbol{k}_{\boldsymbol{0}}$ and $\boldsymbol{k}_{\boldsymbol{a}}$, respectively. The fast, forward-scattered electron (indexed 'a') is detected at the scattered energy $E_{a}=500 \mathrm{eV}$

and at two symmetrical scattering angles, $\theta_{a}=+\left(6^{\circ} \pm 3^{\circ}\right)$ and $-\left(6^{\circ} \pm 3^{\circ}\right)$ as set by input slits at the entrance to the electrostatic lenses associated with the a-toroidal analyser. Throughout this work, positive angles are counted clockwise starting from the incident electron beam direction. Among the two ejected electrons resulting from DI of the target, (labelled ' $b$ ' for the faster and 'c' for the slower), we choose to only detect the faster one, with energy $E_{b}$, in coincidence with the a-scattered electron, hence an (e,3-1e) experiment. Of course, such distinction does not hold in the case of equal ejection energies, but we keep labelling ' $b$ ' the detected electron. These b-electrons are multi-angle analysed in a double toroidal analyser over the angular ranges $\theta_{b}=20^{\circ}-160^{\circ}$ and $200^{\circ}-340^{\circ}$, where $0^{\circ}$ is defined by the incident beam direction. In the off-line analysis, the total $\theta_{b}$-angular range is divided into sectors of width $\Delta \theta_{b}=5^{\circ}$. Though the emission direction of the third, c-electron is unknown, its kinetic 
energy $E_{c}$ is known from the energy conservation $E_{0}-I P^{2+}=E_{a}+E_{b}+E_{c}$, where $I P^{2+}$ is the double ionization potential. [Here, the translational energy of the target atom and the recoil energy imparted to the ion after the emission of the two electrons are neglected, due to the small electron to ion mass ratio]. The present experiments were performed at ejected electron energies corresponding either to an equal energy sharing of $\left(E_{b}: E_{c}\right)=(17: 17) \mathrm{eV}$ or an unequal sharing of $\left(E_{b}: E_{c}\right)=(17: 5) \mathrm{eV} ;(37: 5) \mathrm{eV}$ and $(74: 5) \mathrm{eV}$. The incident energy $\left(E_{0}\right)$ is consequently adjusted to fulfil the energy conservation requirement for the Helium target under study, with $I P^{2+}=79 \mathrm{eV}$. We recall that the previous work published in [29] was obtained with the same $E_{a}$-value of $500 \mathrm{eV}$ and $\left(E_{b}: E_{c}\right)=(51: 10) \mathrm{eV}$, that is under similar enough kinematical conditions (though not exactly identical) to allow meaningful comparison.

Given the above experimental parameters, the momentum transfer to the target, defined by $\boldsymbol{K}=\boldsymbol{k}_{\boldsymbol{0}}-\boldsymbol{k}_{\boldsymbol{a}}$, varies from $K=0.89$ au at $E_{b}+E_{c}=22 \mathrm{eV}$ to $K=1.12 \mathrm{au}$ at $E_{b}+E_{c}=79 \mathrm{eV}$, while, due to the spread in $\theta_{a}$-angle, the momentum transfer resolution amounts to $\Delta K \sim \pm 0.2$ au and the spread in the momentum transfer direction is $\Delta \theta_{K} \sim \pm 10^{\circ}$.

\section{Results and discussion}

Before discussing the He data, we start this Section by recalling that the experimental procedure and the good response of the spectrometer for measuring coincidence angular distributions were validated as described in $[44,45]$. Very briefly, the (e,2e) triple differential cross section (TDCS), $d^{3} \sigma / d E_{a} d \Omega_{a} d \Omega_{b}$, for single ionization of the He 1 s orbital was measured at the energies $E_{a}$ and $E_{b}$ of the current DI experiments on $\mathrm{He}$ and $\mathrm{H}_{2}$, immediately before and after each individual (e,3-1e) run. The so-obtained He TDCS-distributions are in excellent agreement both with the experimental values as well as with the calculated convergent close coupling (CCC) results published in [46-48]. The CCC model is commonly known to produce very accurate TDCS results for electron impact single ionization of He when the residual ion is left in its ground state. Consequently, the invariance of the coincidence collection efficiency upon variation of the $\theta_{b}$-ejected electron angle is ensured.

We now discuss the new DI data. The angular distributions of the (e,3-1e) four-fold differential cross sections (4DCS), $d^{4} \sigma / d E_{a} d E_{b} d \Omega_{a} d \Omega_{b}$, for DI of He at $\left(E_{b}: E_{c}\right)=(17: 5) \mathrm{eV}$ ; $(37: 5) \mathrm{eV} ;(74: 5) \mathrm{eV}$ and $(17: 17) \mathrm{eV}$ are shown in figure $1($ a) to $1(\mathrm{~d})$, respectively. Our 
new data are compared with the calculated results obtained using the very same model which has been used in [43] for DI of He, namely the first Born approximate BBK or 3C model where the final state is described by the product of three Coulomb waves $[42,43]$. Note that the experiments are obtained on a relative scale and have been normalized at the maximum of the forward lobe to the calculations. All the experimental as well as theoretical distributions of the 4DCS display the two-lobe structure well-known for the TDCS: a forward lobe pointing roughly in the momentum transfer direction $(+\boldsymbol{K})$ and a backward lobe pointing in the opposite direction (these two directions are indicated by the vertical dotted lines in the figures). In this sense, we may find some resemblance between the experimental and theoretical distributions. Also, the calculated backward-to-forward maximum intensity ratios are more or less in agreement with the measured ones, with the exception of the $\left(E_{b}: E_{c}\right)=(74: 5) \mathrm{eV}$ case (Fig. 1(c)), where the theory predicts quite a small backward lobe. However, the most striking observations from both figures 1 and 2 are (i) the breaking of symmetry about $\pm \boldsymbol{K}$ directions, (ii) the large shift in the angular position of the lobes, $\sim 30^{\circ}$ to $60^{\circ}$, and (iii) the existence of structures in these lobes. For all energy sharings considered here, the symmetry breaking and the large angular shifts are very much alike those reported in [29] which were obtained at $\left(E_{b}\right.$ : $\left.E_{c}\right)=(51: 10) \mathrm{eV}$ using an older version of our spectrometer. We think that this close resemblance between the 'old' results and the extended new data definitely confirms the validity of our previous results, and should put a final end to the question left open in [39] about this validity. To add one further argument to this statement, we recall that strong nonfirst Born effects were also reported in [12] and in [18], where fully differential (e,3e) cross sections were measured for $\mathrm{He}$ at $E_{0} \sim 600 \mathrm{eV}$ and $\sim 500 \mathrm{eV}$, respectively, that is under kinematics which are similar to the present ones. As stated in the Introduction, second Born calculations [12,37] could only partially account for the observed effects, likely because they include only discrete states as intermediate states and also use the closure approximation. It is not surprising that the strong non-first Born effects observed in (e,3e) cross section distributions are still significantly present in the (e,3-1e) 4DCS, in spite of the integration over the unobserved electron solid angle, $d \Omega_{c}$.

In terms of DI mechanisms, the features observed in Fig. 1 and 2 (i.e. breaking of symmetry, large angular shifts and existence of structures in the measured lobes) cannot be present in any first-order mechanism, namely the SO and TS1 which involve only one single interaction of the projectile with the target. Hence, they are clear signatures of non-first order processes such as TS2 which involves two successive target - projectile interactions. In the absence of second- or higher-order calculations (these are very difficult to perform when using 
the BBK model [49]) which could be compared with our data, we propose hereafter a discussion based on kinematical arguments built on our knowledge of SI (e,2e) processes. This discussion is meant to give a qualitative interpretation of the observed structures as well as an estimate of the angular positions of the lobes, and hence to strengthen the conclusion that the measured strong non-first order effects are not due to an experimental artifact, but are physical effects due to the TS2 mechanism which reveals to play a preponderant role under the present kinematics. For the sake of brevity, we use the data of Fig. 1(c) at $\left(E_{b}: E_{c}\right)=(74$ : 5) $\mathrm{eV}$ and $E_{0}=658 \mathrm{eV}$ for this discussion, but the ideas and the resulting conclusions hold for all the data presented in this work. The discussion is illustrated in figure 2.

TS2 is a two-step process: in the first step (sketched in the top panel of Fig. 2) the slowest c-electron $\left(E_{c}=5 \mathrm{eV}\right)$ is ejected in an (e,2e)-like process where the corresponding scattered electron (called ' $\mathrm{a}^{*}$ ') has an energy $E_{a^{*}}=658-25-5=628 \mathrm{eV}$ (the He SI potential is taken here to be $25 \mathrm{eV}$ ) and appears with the highest probability at the Compton scattering angle, $\pm \theta_{a}$, corresponding to the Bethe ridge condition [50,51]: $E_{0}-E_{a^{*}}=E_{0} \sin ^{2}\left(\theta_{a^{*}}\right)$. This corresponds to $\theta_{a^{*}} \sim \pm 12^{\circ}$. The \pm sign stands for the fact that the intermediate, scattered a*-electron may appear on both sides of the incident beam direction, as shown in figure 2 by the black and red colors, respectively. (Positive angles are here counted clockwise starting from the incident direction). The associated c-electron in this (e,2e) process is most likely ejected in the corresponding momentum transfer direction, that is $\theta_{K^{*}} \sim \pm 78^{\circ}$ with respect to the incident direction. Note that neither the $\mathrm{a}^{*}$ - nor the c-electrons are detected in the $(\mathrm{e}, 3-1 \mathrm{e})$ experiments.

In the second step of TS2, the $\mathrm{a}^{*}$-electron plays the role of the incident projectile in a new $(\mathrm{e}, 2 \mathrm{e})$ ionization of the target, resulting in the pair of electrons effectively detected, that is the a-scattered one with $\left(E_{a}=500 \mathrm{eV}, \theta_{a}=-6^{\circ}\right)$ and the fast b-ejected one with $E_{b}=74 \mathrm{eV}$. Here, two scenarios may occur, depending whether the intermediate $a^{*}$-electron is scattered under $\theta_{a^{*}} \sim+12^{\circ}$ or $-12^{\circ}$. These scenarios are sketched in the middle and bottom panels of Fig. 2, using black and red colors, respectively. We show in the following that they might be responsible, respectively, for the forward and backward lobes observed in the data of Fig. 1.

(1) In scenario 1 (middle panel of Fig. 2), the a-electron is detected at $\theta_{a}=-6^{\circ}$, hence at $18^{\circ}$ from the $\mathrm{a}^{*}$-incident direction. The b-electron is essentially ejected from the target in a binary collision and appears in the corresponding $\theta_{K}$-direction, that is $\theta_{K} \sim+61^{\circ}$ from the $\mathrm{a}$ *-incident direction (at the high energies involved here, the recoil intensity appearing about $-\boldsymbol{K}$ direction is quite small [52]). In other words, the b-electron is mostly ejected at $\theta_{b} \sim+\left(61^{\circ}+12^{\circ}\right)=+73^{\circ}$ from the primary incident beam direction. In the experimental 
data displayed in Fig. 1(c), the forward lobe is peaking at about $70^{\circ}$. On the one hand, this value is quite far from the momentum transfer direction $\theta_{K} \sim+34^{\circ}$ where the firstorder SO and TS1 contributions should be at their maximum. But on the other hand, this value is quite close to $+73^{\circ}$ derived from the above scenario 1 . We thus conclude that the shift of the forward lobe in Fig. 1(c) is essentially due to the TS2 contribution, according to this scenario 1. SO and TS1 contributions might of course be also present, but their intensity is appreciably smaller than that of the TS2.

(2) In scenario 2 (bottom panel of Fig. 2), the a-electron is again detected at $\theta_{a}=-6^{\circ}$, but now this corresponds to $+6^{\circ}$ from the intermediate $\mathrm{a}^{*}$-incident direction. The b-electron ejected from the target appears essentially in the corresponding $\theta_{K}$-direction, that is $\theta_{K} \sim$ $-40^{\circ}$ from the $\mathrm{a}^{*}$-incident direction (here also, the recoil intensity appearing about $-\boldsymbol{K}$ direction is quite small). In other words, the b-electron is mostly ejected at $\theta_{b} \sim$ $\left(40^{\circ}+12^{\circ}\right)=-52^{\circ}$, or alternatively at $\theta_{b} \sim\left(360^{\circ}-52^{\circ}\right)=308^{\circ}$ from the primary incident beam direction. In the data displayed in Fig. 1(c), the backward lobe is peaking at $\sim 310^{\circ}$. On the one hand, this value is quite far from the $-K$ direction, $\theta_{-K}=180^{\circ}+34^{\circ}=214^{\circ}$ where the first-order SO and TS1 contributions should be at their maximum. But on the other hand, this value is in excellent agreement with the value $+308^{\circ}$ derived from scenario 2. We thus again conclude that the backward lobe position in Fig. 1(c) is essentially determined by the TS2 contribution, according to this scenario 2. Once more, SO and TS1 contributions are appreciably smaller than that of TS2.

This discussion and the resulting conclusions may be likewise repeated for all the data presented in figure 1. We may add one further observation: each of the experimental forward as well as backward lobes in Fig. 1 appears to be split in two sub-lobes. From the discussion above, the sub-lobes located at the largest angles can be attributed to the TS2 contribution, whereas the ones located at smallest angles are grossly peaking at $\boldsymbol{+} \boldsymbol{K}$ or $\boldsymbol{- K}$ direction, and hence may be attributed to the first-order SO and/or TS1 contributions.

This splitting in two sub-lobes is most obvious in Fig. 1(b), but it can still be clearly noticed in all the data. We also note (at least for the backward sub-lobes) that the relative importance of the two sub-lobes strongly changes with the $\left(E_{b}: E_{c}\right)$ energy sharing, the maximum intensity of the second-order sub-lobe being respectively $\sim 20 \%$ smaller, $\sim 25 \%$ larger and much larger ( 2 to 3 times larger) than the maximum intensity of the first-order sublobe, when $E_{b}$ is increased from 17 to 37 and to $74 \mathrm{eV}$ at constant $E_{c}=5 \mathrm{eV}$ (Fig. 1 (a) to(c)). This means that for the kinematics considered here, the TS2 contribution becomes more and 
more preponderant with increasing $E_{b}$ and is dominant over the SO and TS1 ones. Such dominance confirms the observations made in previously published (e,3e) [12] and (e,3-1e) [29] experiments at about the same incident energy. This makes it clear that the introduction of beyond first-order terms of the Born series in any future theoretical work is necessary at the intermediate electron impact regime investigated here.

\section{Conclusion}

We have performed new relative (e,3-1e) experiments for double ionization of $\mathrm{He}$ at $600 \mathrm{eV}$ impact energy and for different energy-sharings $\left(E_{b}: E_{c}\right)$. Our data display large shifts of the forward and backward lobes in the ejected electron angular distributions, thus confirming and validating the observations made earlier in [29], and consequently removing the questioning put in [39] on these earlier results. We also present a qualitative analysis based on kinematical arguments which shows that under the present kinematics the two-step 2 (TS2) mechanism dominates over the SO and the TS1 and is mostly responsible for the structures and angular positions of the measured lobes.

\section{Ackowledgments}

A N and P B wish to acknowledge the Université de Paris-Sud 11 for an 'ATER position' and a 'Professeur invité position', respectively. 


\section{References}

[1] Lahmam-Bennani A, Dupré C and Duguet A 1989 Phys. Rev. Lett. 631582

[2] Lahmam-Bennani A 1991 J. Phys. B: At. Mol. Opt. Phys. 242401

[3] Lahmam-Bennani A, Duguet A, Grisogono A M and Lecas M 1992 J. Phys. B: At. Mol. Opt. Phys. 252873

[4] El Marji B, Schröter C, Duguet A, Lahmam-Bennani A, Lecas M and Spielberger L1997 J. Phys. B: At. Mol. Opt. Phys. 303677

[5] Schröter C, El Marji B, Lahmam-Bennani A, Duguet A, Lecas M and Spielberger L 1998 J. Phys. B: At. Mol. Opt. Phys. 31131

[6] Taouil I, Lahmam-Bennani A, Duguet A and Avaldi L1998 Phys. Rev. Lett. 814600

[7] Lahmam-Bennani A, Taouil I, Duguet A, Lecas M, Avaldi L and Berakdar J 1999 Phys. Rev. A 593548

[8] Kheifets A, Bray I, Duguet A, Lahmam-Bennani A and Taouil I 1999 J. Phys. B: At. Mol. Opt. Phys. 325047

[9] Lahmam-Bennani A, Duguet A, Gaboriaud M N, Taouil I, Lecas M, Kheifets A, Berakdar J and Dal Cappello C 2001 J. Phys. B: At. Mol. Opt. Phys. 343073

[10] Jia C C, Lahmam-Bennani A, Duguet A, Avaldi L, Lecas M and Dal Cappello C 2002 J. Phys. B: At. Mol. Opt. Phys. 343073

[11] Lahmam-Bennani A, Jia C C, Duguet A and Avaldi L 2002 J. Phys. B: At. Mol. Opt. Phys. 35 L215

[12] Lahmam-Bennani A, Duguet A, Dal Cappello C, Nebdi H and Piraux B 2003 Phys. Rev. A 67 010701(R)

[13] Berakdar J, Lahmam-Bennani A and Dal Cappello C 2003 Physics Reports 374 91-164

[14] Jia C C, Lahmam-Bennani A, Dal Cappello C, Duguet A and Avaldi L 2003 J. Phys. B: At. Mol. Opt. Phys. 36 L17

[15] Dorn A, Mosshammer R, Schröter C D, Zouros T J M, Schmitt W, Kollmus H, Mann R and Ullrich J 1999 Phys. Rev. Lett. 822496

[16] Dorn A, Kheifets A, Schröter C D, Najjari B, Höhr C, Mosshammer R and Ullrich J 2001 Phys. Rev. Lett. 863755

[17] Ullrich J, Mosshammer R, Dorn A, Dörner R, Schmidt L Ph H and Schmidt-Böcking H 2003 Rep. Prog. Phys. 66 1463-1545

[18] Dorn A, Kheifets A, Schröter C D, Höhr C, Sakhelashvili G, Mosshammer R,Lower J and Ullrich J 2003 Phys. Rev. A 68 012715; see also 'Comment' by Lahmam-Bennani A, Catoire F, Duguet A and Dal Cappello C 2005 Phys. Rev. A 71 026701; and 'Reply to Comment' by Dorn A et al 2005 Phys. Rev. A 71026702

[19] Dürr M, Dorn A, Ullrich J, Cao S P, Czasch A, Kheifets A S, Götz J R and Briggs J S. 2007 Phys. Rev. Lett. 98193201

[20] Ren X G, Dorn A and Ullrich J. 2008 Phys. Rev. Lett. 101093201

[21] Cao S P, Ma X, Dorn A, Dürr M and Ullrich J.2008 Phys. Rev. A 77062703

[22] Ford M J, Doering J P, Coplan M A, Cooper J W and Moore J H 1995 Phys. Rev. A 51 418

[23] Dupré C, Lahmam-Bennani A and Duguet A 1991 Meas. Sci. Technol. 2327

[24] Duguet A, Dupré C and Lahmam-Bennani A 1991 J. Phys. B: At. Mol. Opt. Phys. 24675

[25] Lahmam-Bennani A, Ehrhardt H, Dupré C and Duguet A 1991 J. Phys. B: At. Mol. Opt. Phys. 243645

[26] Duguet A and Lahmam-Bennani A 1992 Z Phys D: At., Mol. Clusters 23383

[27] El Marji B, Duguet A, Lahmam-Bennani A, Lecas M and Wellenstein H F 1995 J. Phys. B: At. Mol. Opt. Phys. 28 L733 
[28] El Marji, Lahmam-Bennani A, Duguet A and Reddish T J 1996 J. Phys. B: At. Mol. Opt. Phys. 29 L157

[29] Lahmam-Bennani A, Duguet A and Roussin S 2002 J. Phys. B: At. Mol. Opt. Phys. 35 L59

[30] Watanabe N, Khajuria Y, Takahashi M, Udagawa Y, Vinitsky P S, Popov Yu V, Chuluunbaatar O and Kouzakov K A 2005 Phys. Rev. A 72032705

[31] Ford M J, El Marji B, Doering J P, Moore J H, Coplan M A and Cooper J W 1998 Phys. Rev. A 57325

[32] Carlson T A and Krause M O 1965 Phys Rev 1401057

[33] Tweed R J 1992 Z Phys D: At., Mol. Clusters 23309

[34] LeRouzo H and Dal Cappello C 1991 Phys. Rev. A 43318

[35] Dal Cappello C and LeRouzo H 1991 Phys. Rev. A 431395

[36] McGuire J H 1982 Phys. Rev. Lett. 491153

[37] Mansouri A, Dal Cappello C, Houamer S, Charpentier I and Lahmam-Bennani A $2004 J$. Phys. B: At. Mol. Opt. Phys. 371203

[38] Serov V V and Joulakian B B 2009 Phys. Rev. A 80062713

[39] Götz J R, Walter M and Briggs J S 2003 J. Phys. B: At. Mol. Opt. Phys. 36 L77

[40] Götz J R, Walter M and Briggs J S 2006 J. Phys. B: At. Mol. Opt. Phys. 394365

[41] Catoire F, Staicu Casagrande E M, Lahmam-Bennani A, Duguet A, Naja A, Ren X G, Lohmann B and Avaldi L 2007 Rev. Sci. Instrum. 78013108

[42] Joulakian B, Dal Cappello C and Brauner M 1992 J. Phys. B: At. Mol. Opt. Phys. 25 2863

[43] Ancarani L U, Gasaneo G, Colavecchia F D and Dal Cappello C 2008 Phys. Rev. A 77 062712

[44] Naja A, Staicu Casagrande E M, Lahmam-Bennani A, Nekkab M, Mezdari F, Joulakian B, Chuluunbaatar O and Madison D H 2007 J. Phys. B: At. Mol. Opt. Phys. 403775

[45] Lahmam-Bennani A, Naja A, Staicu Casagrande E M, Okumus N, Dal Cappello C, Charpentier I and Houamer S 2009 J. Phys. B: At. Mol. Opt. Phys. 42165201

[46] Staicu Casagrande E M, Naja A, Mezdari F, Lahmam-Bennani A, Bolognesi P, Joulakian B, Chuluunbaatar O, Al-Hagan O, Madison D H, Fursa D V and Bray I 2008 J. Phys. B: At. Mol. Opt. Phys. 41025204

[47] Kheifets A S, Naja A, Staicu Casagrande E M and Lahmam-Bennani A 2008 J. Phys. B: At. Mol. Opt. Phys. 41 145201; ibid 200841209801

[48] Kheifets A S, Naja A, Staicu Casagrande E M and Lahmam-Bennani A 2009 J. Phys. B: At. Mol. Opt. Phys. 42165204

[49] Ancarani L U, Montagnese T and Dal Cappello C 2004, in 'Electron and Photon Impact Ionisation and Related Topics',vol.183 p21-31, IOP, edited by B. Piraux

[50] Inokuti M 1971 Rev. Mod. Phys.43 297

[51] Lahmam-Bennani A, Wellenstein H F, Dal Cappello C, Duguet A and Rouault M $1983 \mathrm{~J}$. Phys. B 16, 2219

[52] Avaldi L, Camilloni R, Fainelli E, Stefani G, Franz A, Klar H and McCarthy I E 1987 J. Phys. B: At. Mol. Phys. 205827 


\section{Caption to figures}

Figure 1 (Color online): Four-fold differential cross sections (4DCS) for double ionization of He. The scattered electron with energy $E_{a}=500 \mathrm{eV}$ is detected at an angle $\theta_{a}=-6^{\circ}$ in coincidence with the fast-emitted electron with energy $E_{b}$, whereas the slow-emitted electron with energy $E_{c}$ remains undetected. Panel (a): $\left(E_{b}: E_{c}\right)=(17: 5) \mathrm{eV},(\mathrm{b}):\left(E_{b}: E_{c}\right)=(37: 5)$ $\mathrm{eV}$, (c) $\left(E_{b}: E_{c}\right)=(74: 5) \mathrm{eV}$ and $(\mathrm{d}):\left(E_{b}: E_{c}\right)=(17: 17) \mathrm{eV}$. The incident electron energy $E_{0}$ is adjusted to fulfil the energy conservation requirement for DI of He. Full dots are the experimental data, with one standard deviation statistical error bar. Solid curve: theoretical predictions from the first-order BBK model. The absolute scale shown is that of theory, to which the experiment is normalised for best visual fit at the maximum of the forward lobe. The thin dotted lines indicate the direction of the momentum transfer $\left(\theta_{K}\right)$ and its opposite.

Figure 2 (Color online): Sketch of the kinematics of a two-step 2 (TS2) process, in the case of $\left(E_{b}: E_{c}\right)=(74: 5) \mathrm{eV}$. Top panel shows the first step interaction with the target, whereas the two bottom panels show the second step. Middle panel (black colour) stands for the case

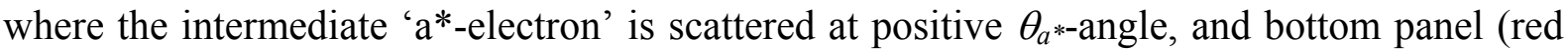

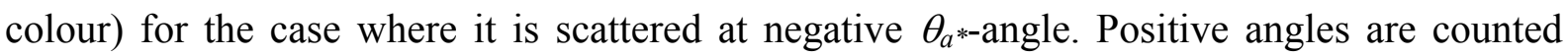
clockwise starting from the incident direction. See text for more explanations. 

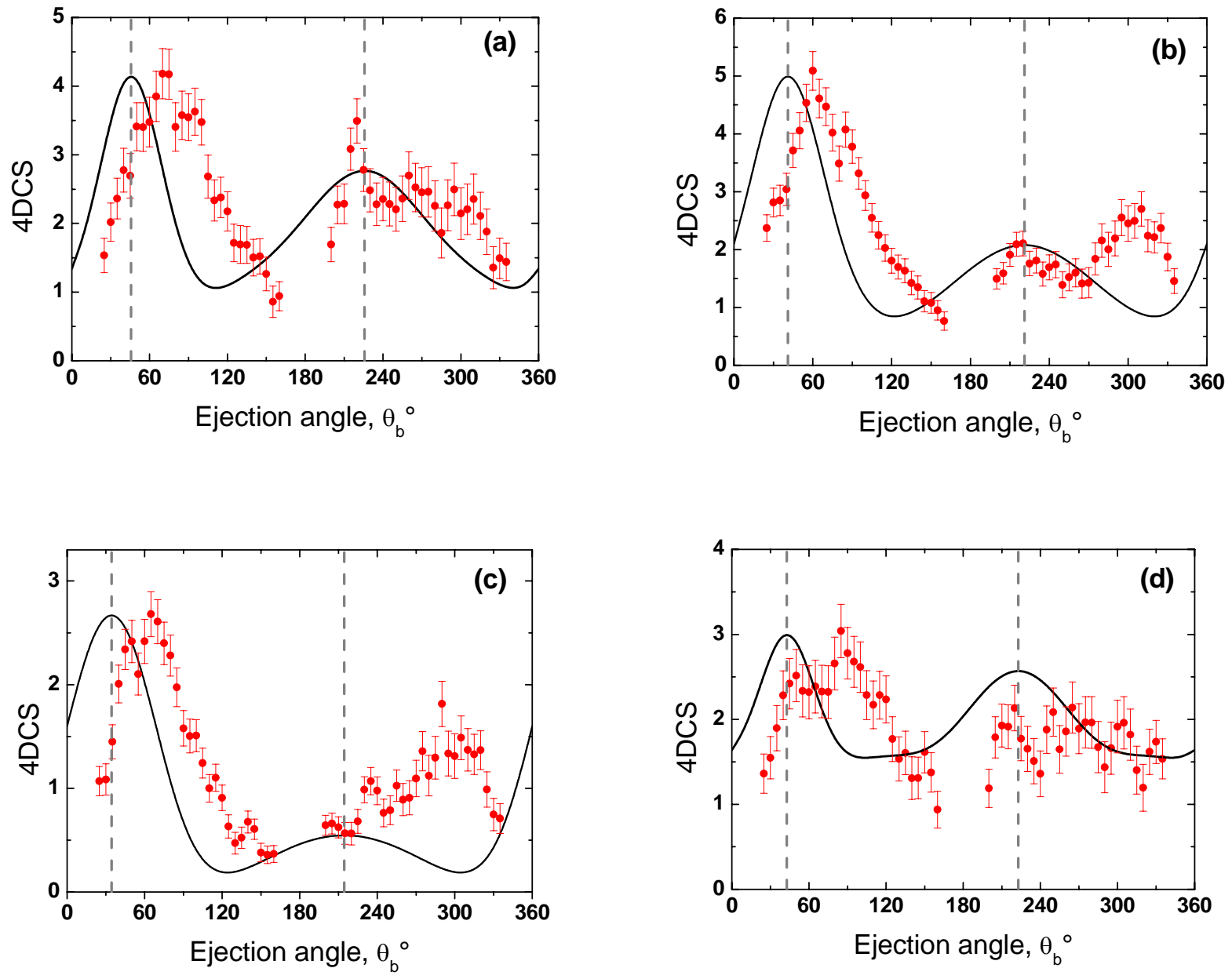

Lahmam-Bennani A et al

Figure 1 


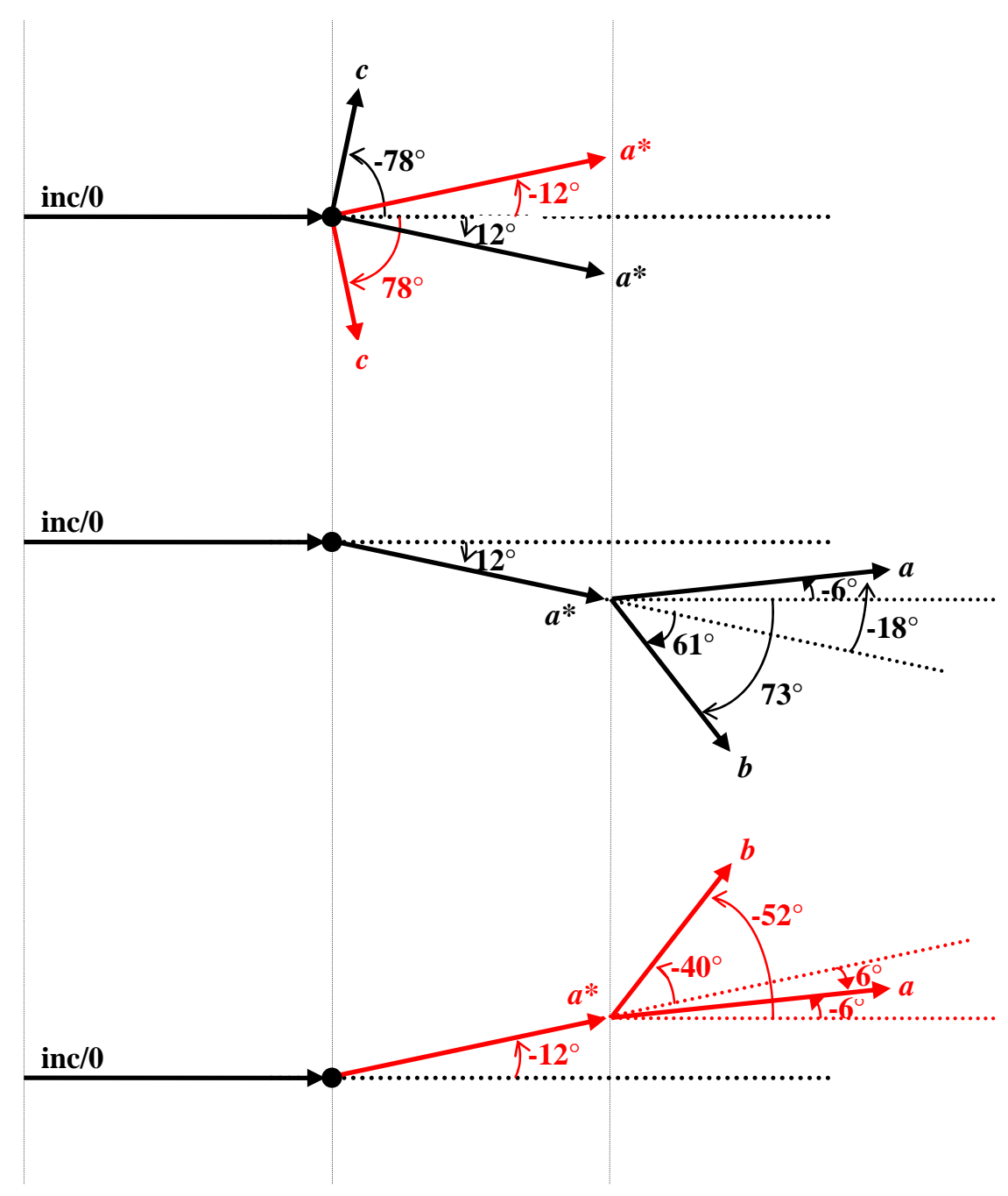

Lahmam-Bennani A et al

Figure 2 